\title{
Laser Ionization Studies of Gas Phase and Surface Adsorbed Molecules
}

\author{
JON T. MEEK, S. RANDOLPH LONG, RICHARD B. OPSAL and \\ JAMES P. REILLY†
}

Department of Chemistry, Indiana University, Bloomington, Indiana, 47405

Gas phase hydrocarbons are ionized using the output of UV excimer lasers and frequency doubled dye lasers. The ions generated are mass resolved and kinetic energy distributions of ejected photoelectrons are recorded. Information about the photochemistry and ion spectroscopy of benzaldehyde, benzene and toluene is derived. Molecules adsorbed to metal surfaces are also laser ionizable leading to distinctively sharp peaks in their laser induced time of flight mass spectra.

While the earliest studies of laser induced ionization were performed in the mid 1960s, ${ }^{1,2}$ this field of research has developed and expanded particularly rapidly in just the last seven years. The acceleration is undoubtedly attributable to such exciting developments as single atom detection using dye lasers ${ }^{3}$ and the application of multiphoton ionization to problems in atomic ${ }^{4-6}$ and molecular spectroscopy. ${ }^{7-9}$ Mass spectrometry based on a laser ionization source has been discussed for over a decade, ${ }^{10}$ but many of the exciting developments in this area have occurred in just the last few years. ${ }^{11-16}$ The evolution of commercial laser systems has played a major role in this recent progress. For laser ionization to have a significant impact on mass spectrometry, the ionization process must be efficient. Intense pulsed light sources of high energy photons are therefore needed in order to efficiently induce stepwise two photon ionization. Excimer lasers

$\dagger$ Alfred P. Sloan Fellow. 
and powerful frequency doubled dye lasers which satisfy this requirement have only been commercially available for a few years.

When considered superficially, the detection of very small concentrations of molecules by laser ionization mass spectrometry might be viewed as a simple extension of previous successful experiments with alkali atoms. ${ }^{3}$ However, the molecular situation is actually considerably more complicated. First of all, absorption cross sections of molecules are much lower than those of atomic resonance lines. Second, molecular ionization potentials are considerably higher and rarely can be reached with a pair of conveniently available visible dye laser photons. Third, molecules undergo photophysical and photochemical processes such as intersystem crossing, predissociation and isomerization which atoms do not. Since these processes occur following absorption of one photon, they may compete with the second step of a two photon ionization process and thereby reduce the observed ion yield. Finally, because the laser wavelengths required to reach molecular ionization potentials in two steps are so short, background ionization is a major problem which interferes with the detection of low concentrations of hydrocarbons. In spite of these obstacles, we have recently demonstrated multi-femtogram sensitivity for detecting gas chromatograph effluent and selectivity capable of resolving $\mathrm{C}_{18} \mathrm{H}_{12}$ isomers which cannot be distinguished by either conventional capillary gas chromatography or mass spectrometry. ${ }^{17}$

While someone concerned purely with analytical detection sensitivity would view excited state molecular photochemistry as simply an annoying interference to be avoided when designing an efficient laser ionization process, the problem could clearly be turned around and considered from another perspective, i.e., laser induced ion yields must convey information about interfering photochemical processes which should be extractable by someone appropriately motivated. We have recently found this to be the case in a study of the laser ionization mass spectrum of benzaldehyde vapor. ${ }^{18}$ Using a $\mathrm{KrF}$ laser as photolysis source we found the benzaldehyde mass spectrum to be virtually identical to that of gas phase benzene, with only a hint of the molecular ion at $m / e=106$. Naturally the question which arises is whether benzaldehyde ionizes and then fragments or, alternatively, does it absorb one photon, fragment to benzene, then ionize? The key to resolving this issue is found in the laser photoelectron spectrum of benzaldehyde. By monitoring the kinetic energies of the electrons 
ejected from the ionizing gas we hoped to be able to determine which of the molecules was in fact actually ionizing. The result of our study is that the benzaldehyde and benzene laser photoelectron spectra are identical, indicating that benzaldehyde in fact rearranges to benzene before being ionized. The ten nanosecond time resolution of our pulsed laser is insufficient to resolve this fast kinetic process, but it seems likely that with picosecond ultraviolet laser pulses the time dependence of the collision-free production of benzene from benzaldehyde should be observable. With ionizing pulses which are sufficiently short and intense, $\mathrm{C}_{6} \mathrm{H}_{6}^{+}$ions should not be generated from pure benzaldehyde, since the parent ion at $m / e=106$ is known to be stable from electron impact mass spectra. ${ }^{19}$

Another example of how photoelectron studies are of potentially great interest in sorting out the mechanism of a laser ionization process is the laser induced ionization of benzene by $\mathrm{KrF}$ and $\mathrm{ArF}$ excimer lasers. In the first successful laser photoelectron spectroscopy experiment which we performed ${ }^{20}$ we showed that the $\operatorname{KrF}(2485 \AA)$ induced spectrum, which shows a single broad peak (see Figure 1) can be interpreted as a simple two step ionization process without complicating intermediate state effects. However the ArF (1930 $\AA$ ) induced spectrum, which is also displayed in Figure 1, exhibits two peaks. The second, broader peak decreases relative to the first as the light intensity increases. The first peak corresponds to simple two step ionization of benzene with the electrons carrying off most of the deficit between the photon input energy $(12.8 \mathrm{eV})$ and the ionization potential $(9.25 \mathrm{eV})$. The second peak apparently results from the ionization of some state of benzene formed following single photon absorption. This might be a triplet or isomer state. The benzene ion must be generated in a different geometry or vibrational state distribution, compared with when it is ionized by $\mathrm{KrF}$ laser radiation, in order to generate the slow electrons which appear in the second ArFinduced photoelectron peak. Any hope for assigning the excited states involved will require electron energy resolution improvements. Recent developments in this area are described next.

In order to resolve vibrational structure which is obviously masked under the broad photoelectron profiles of Figure 1 (the peaks of which are about $500 \mathrm{meV}$ wide), we switched from a spectrally broad, relatively long time duration excimer laser to a narrow band short pulse dye laser photolysis source. The resulting improvements in 

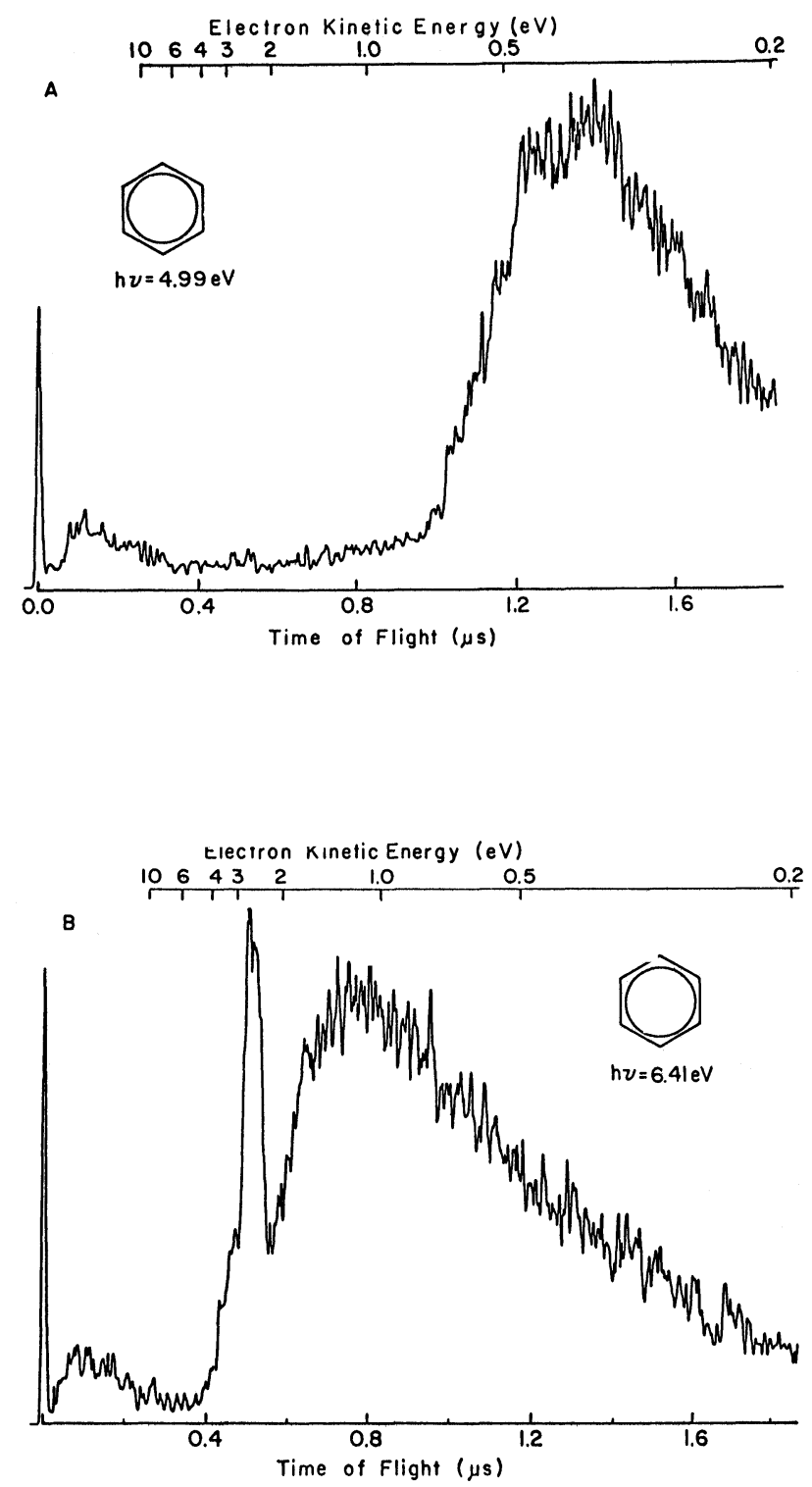

FIGURE 1 Time of flight photoelectron spectra of benzene ionized by radiation from (b) $\mathrm{KrF}$ (b) ArF excimer lasers. 
photoelectron energy resolution are drastic, as can be seen in Figures 2 and 3. Resolution is now about $7 \mathrm{meV}$ (approximately $60 \mathrm{~cm}^{-1}$ ) and vibrational structure under the photoelectron band is easily resolved. Most interestingly, the photoelectron energy distribution depends strongly on the vibronic intermediate state excited by the frequency doubled dye laser. Several vibrational states within the ${ }^{1} B_{2 u}$ first excited singlet state of benzene have been utilized. When the laser is tuned to the $6_{1}^{0}$ transition, ions are formed predominantly vibrationally unexcited. When tuned to the $6{ }_{1}^{0} 16_{1}^{1}$ transition however, virtually all ions are generated with one quantum of excitation in $\nu_{16}$.

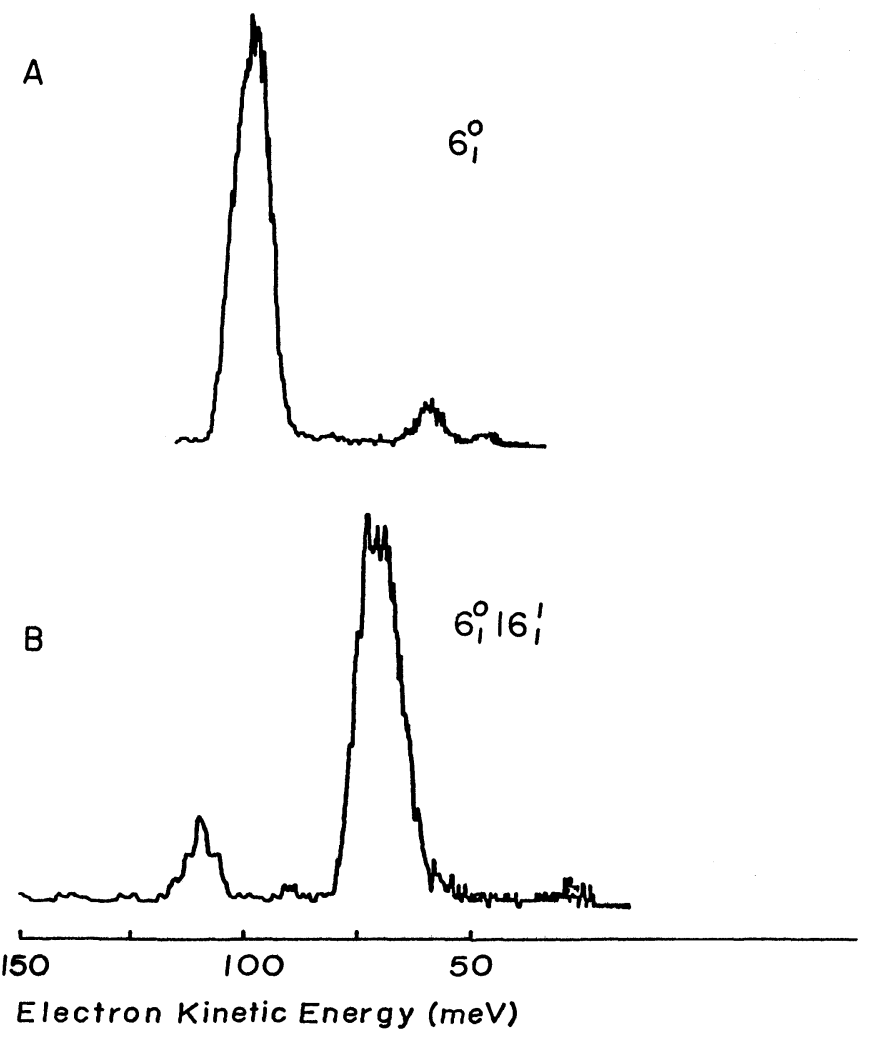

FIGURE 2 Kinetic energy distribution of the photoelectrons ejected from benzene ionized by the output from a narrowband frequency doubled dye laser. The radiation is tuned to the (a) $6_{1}^{0}$ (b) $6_{1}^{0} 16_{1}^{1}$ vibronic transitions in the ${ }^{1} B_{2 u} \leftarrow{ }^{1} A_{1 \mathrm{~g}}$ absorption band system of $\mathrm{C}_{6} \mathrm{H}_{6}$. 


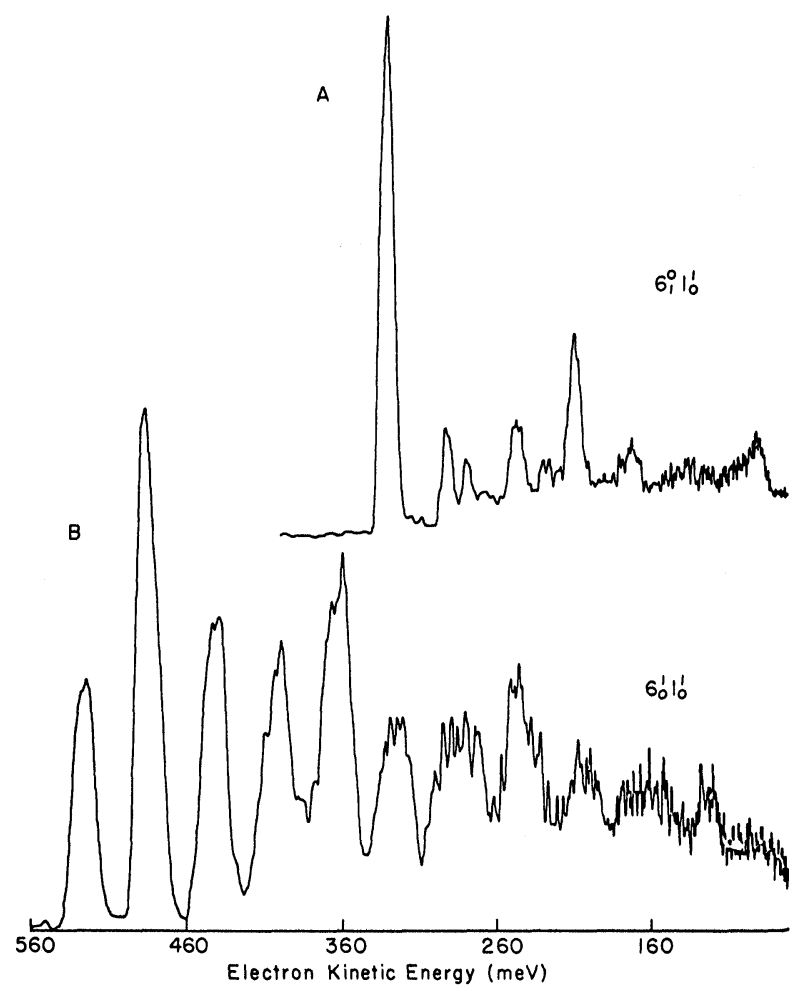

FIGURE 3 Same as Figure 2 except that the vibronic transitions involved are (a) $6_{1}^{0} 1_{0}^{1}$ and (b) $60_{0}^{1} 1_{0}^{1}$.

(The assignment of this ion peak, which has never been observed in a conventional photoelectron spectrum of benzene, follows from three mutually conclusive bits of data: First, the vibronic state from which the benzene ionizes in this experiment has been previously assigned as $v=1$ of $\nu_{16 .}{ }^{21}$ Second, in the Rydberg spectrum of benzene a vibration of comparable frequency has been assigned to $\nu_{16 .}{ }^{22}$ Third, isotope substitution experiments which we have performed with benzene yield the correct frequency ratio.) When the transition $6{ }_{1}^{0} 1_{0}^{1}$ is excited, $v=0$ ions are preferentially formed, but the second most populated vibrational state is $122 \mathrm{meV}$ above the ground vibrational state. This peak appears in the conventional photoelectron spectrum of benzene and has been previously assigned to $v=1$ of $\nu_{1}{ }^{23,24}$ 
Considering the vibronic state which we pass through to obtain this spectrum, this work represents strong corroboration of the earlier assignment. Finally, when the transition $6{ }_{0}^{1} 1_{0}^{1}$ is excited, a photoelectron spectrum with 12 peaks is generated as displayed in Figure 3. This is quite remarkable considering that the conventional single photon photoelectron spectrum of benzene contains only four or five peaks. More ion vibrational states can apparently be accessed when benzene is photoionized from a vibrationally excited $S_{1}$ state rather than from the vibrationless $S_{0}$ ground state. The Franck-Condon principle can be invoked to rationalize the observed trends, although calculations of the potential energy surfaces of the neutral and ion electronic states will be required to quantitatively interpret relative peak heights. A complete analysis of the benzene data is being prepared for publication. ${ }^{25} \mathrm{We}$ have recently obtained and published analogous results for toluene. ${ }^{26}$ Similar vibronic state dependences are evident. In summary, laser photoelectron spectra contain information about what species is ionizing, how many photons are required to induce ionization and what the vibrational frequencies and state distribution of the product ions are.

A very recent development in our work in this area has been the observation of laser induced ionization of hydrocarbon molecules absorbed to metal surfaces. We originally observed this with gas phase benzene which was being sprayed onto a Ni grid. Similar results have now been obtained with heavy polyaromatic hydrocarbons. Briefly, when a UV light pulse is focussed into the interaction/ionization region of our laser mass spectrometer, depicted in Figure 4, ions are formed in the gas phase by two step ionization. A TOF mass spectrum of benzene is displayed in Figure 5a (at low intensities, only a single peak at mass 78 is observed). As the laser beam is shifted towards a metal surface in the ionization region, a second, much narrower peak appears in the mass spectrum, as seen in Figures $5 \mathrm{~b}-5 \mathrm{~d}$. This peak results from ionization of benzene molecules at the surface. This hypothesis was confirmed in the following ways:

1) The peak appears only when the laser focussing lens is moved so as to illuminate the region near grid 0 and it disappears when the lens moves back toward grid 1 .

2) A simple computer model which calculates ion flight times based on their mass, initial velocity and position and fragmentation dynamics 

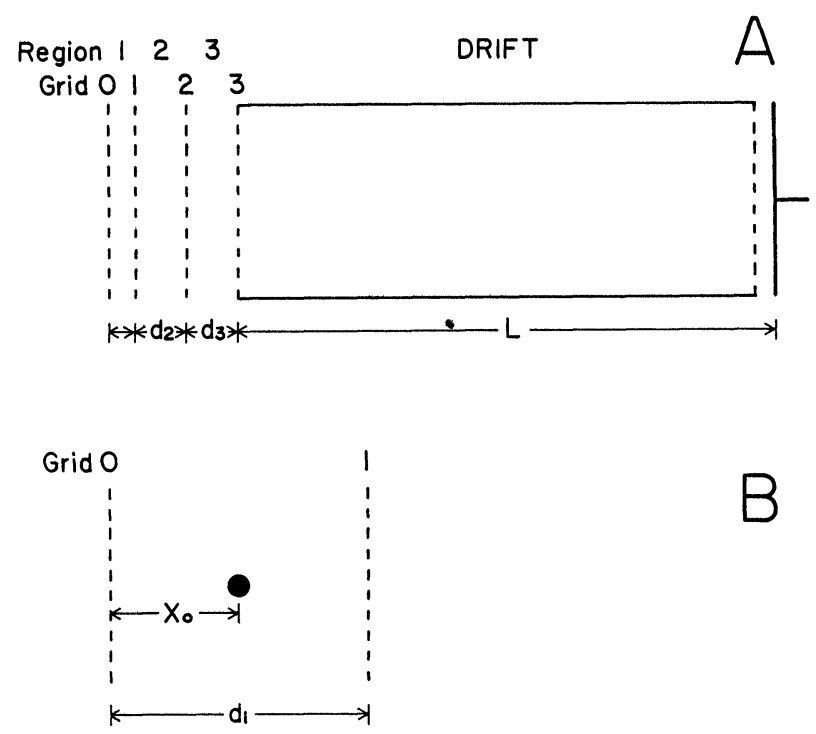

FIGURE 4 Basic ionization region and flight tube design of our home-made laser TOF mass spectrometer.

accurately predicts the location of the sharp peak only if we assume that the ions originate at the surface, not further to the right in the gas phase.

3) Shifting the lens position moves the laser focal spot around within region 1 . When this is done the broad (gas phase) ion peak shifts in time, but the narrow (surface) peak, when it appears, always appears at precisely the same time. These dependences on lens position are exactly what one would expect for ion peaks resulting from gas phase and surface ionization.

4) The above data were recorded with +2000 volts on grid 0 and -2000 volts on grids 1,2 and 3 . Setting the voltage on grid 2 to $+1950 \mathrm{~V}$ should prevent passage of all ions except those formed within 75 microns of grid 0 . When this is experimentally tested the broad peak disappears from the mass spectrum but the sharp peak remains. Details of the ionization mechanism remain to be established, but electron transfer may be occurring from an excited absorbed molecule to the metal surface. Whether or not this phenomenon will 


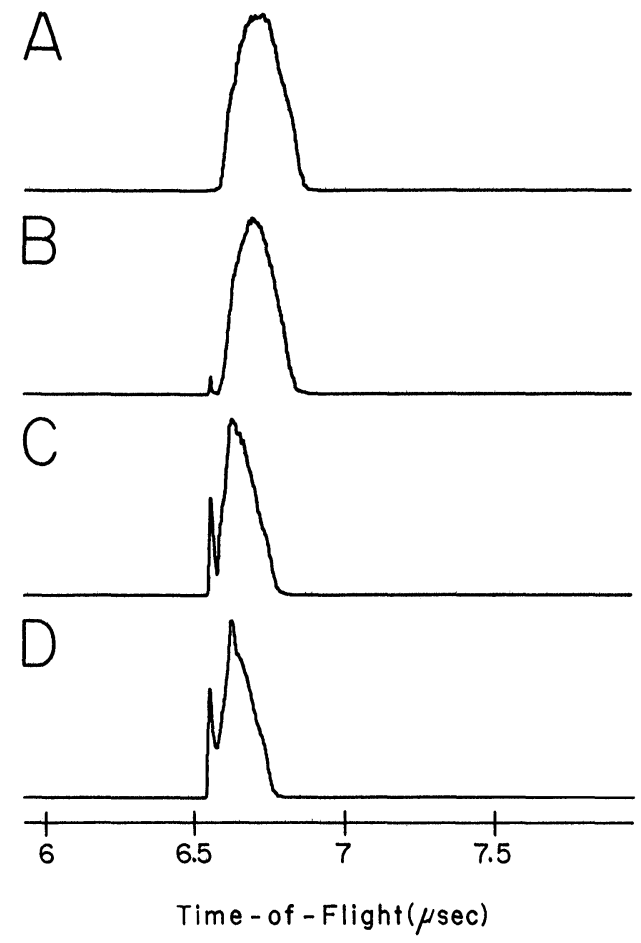

FIGURE 5 TOF mass spectra of benzene ionized with low intensity $\mathrm{KrF}$ laser radiation. Only the region near $m / e=78$ is displayed. In (a) the light irradiates gas phase molecules between grids 0 and 1 (see Figure 4). In (b) $\rightarrow$ (d) the laser focal spot is moved progressively closer to grid 0 .

enable us to record electronic spectrum of surface adsorbed species simply by tuning the laser wavelength depends on the exact ionization mechanism and at the present time remains to be seen. Due to the narrowness of the mass spectral ion peaks, we are currently interested in the potential of this technique as a method of sample introduction in mass spectrometry.

\section{Acknowledgements}

This work has been supported by the US Army Research Office under contract number DAAG29-81-K-0097 and by the Office of Research and Development, Environmental Protection Agency, under grant number R808790-01-0. 


\section{References}

1. F. V. Bunkin and A. M. Prokhorov, Sov. Phys. JETP 19, 739 (1964); L. V. Keldish, Zh. Eksp. Teor. Fiz. 47, 1945 (1964).

2. G. S. Voronov and N. B. Delone, Pis ' $m a$ Zh. Eksp. Teor. Fiz. 1, 42 (1965).

3. (a) G. S. Hurst, M. H. Nayfeh and J. P. Young, Appl. Phys. Lett. 30, 229 (1977); (b) G. S. Hurst et al., Rev. Mod. Phys. 51, 767 (1979).

4. D. Popescu, C. B. Collins, B. W. Johnson and I. Popescu, Phys. Rev. A 9, 1182 (1974).

5. P. Eshenck, J. A. Armstrong, R. W. Dreyfus and J. J. Wynne, Phys. Rev. Lett. 36, 1296 (1976).

6. N. D. V. Bohm, W. Michaelis and C. Weitkomp, Optics Comm. 26, 177 (1978).

7. P. M. Johnson, Acc. Chem. Res. 13, 20 (1980).

8. D. H. Parker, J. O. Berg and M. A. El-Sayed, in: Advances in Laser Chemistry, Springer Series on Chemical Physics, Vol. 3, ed. A. H. Zewail (Springer-Verlag, Berlin, 1978).

9. T. G. Dietz, M. A. Duncan and R. E. Smalley, J. Chem. Phys. 76, 1227 (1982).

10. R. V. Ambartsumayn and V. S. Letokhov, Appl. Opt. 11, 354 (1972).

11. V. S. Letokhov, Phys. Today 30, 23 (1977).

12. S. Rockwood, J. P. Reilly, K. Hohla and K. L. Kompa, Opt. Comm. 28, 175 (1979).

13. J. P. Reilly and K. L. Kompa, J. Chem. Phys. 73, 5468 (1980).

14. L. Zandee and R. B. Bernstein, J. Chem. Phys. 71, 1359 (1979).

15. U. Bosel, H. J. Neusser and E. W. Schlag, J. Chem. Phys. 72, 4327 (1980).

16. D. M. Lubman, R. Naaman and R. N. Zare, J. Chem. Phys. 72, 3034 (1980).

17. G. Rhodes, R. B. Opsal, J. T. Meek and J. P. Reilly, Anal. Chem. 55, 280 (1983).

18. S. R. Long, J. T. Meek, P. J. Harrington and J. P. Reilly, J. Chem. Phys. 78, 3341 (1983).

19. E. Stehagen, S. Abrahamsson, F. W. McLafferty, Registry of Mass Spectral Data (Wiley, New York, 1974).

20. J. T. Meek, R. K. Jones and J. P. Reilly, J. Chem. Phys. 73, 3503 (1980).

21. G. H. Atkinson and C. S. Parmenter, J. Molec. Spectros. 73, 31 (1978).

22. P. G. Wilkinson, Canad. J. Phys. 34, 608 (1956).

23. L. Asbrink, E. Lindholm and O. Edqvist, Chem. Phys. Lett. 5, 609 (1970).

24. A. W. Potts, W. C. Price, D. G. Streets and T. A. Williams, Faraday Disc. Chem. Soc. 54, 168 (1972).

25. S. R. Long, J. T. Meek and J. P. Reilly (submitted to J. Chem. Phys.).

26. J. T. Meek, S. R. Long and J. P. Reilly, J. Phys. Chem. 86, 2809 (1982). 